\title{
SIEBEN STELLEN DEUTSCH FÜR DIE E.O.I.
}

W

ie allgemein bekannt, hat die Junta de Andalucia dieses Jahr tatsächlich sieben Deutschstellen für die EEOOll ausgeschrieben. Sieben, eine magische Zahl, wie die Sieben Weltwunder. Im März wird die Ausschreibung im BOJA veröffentlicht, und im Juli wird es dann wieder für einige so weit sein, das mühsame Auswahlverfahren durchzumachen.

Ich lerve Dentsch weil meine Mutter ist Deutsche vicht and sie Weipt Kein Dentschuort, weil derentschy ist micht wichtig fir meine zukunft (ich glaube das), und weil itt brauche ich dex Dentsch nicht fuir meinen Beruf. Jeh studiere. Deutschweil bin ich "masoquista" und das mich.

Auperdem Deutsch nicht sprechen ich spreche ein bipchen Frau zösich (un petit pen") und ich lese den Wissenschaflichenglisch. Wänn reise ion, irusuer sage wich warum sprechen die Spanier so went Freudsprachen? Ist es weil wir hadengehr schlecht Politiker? $\rightarrow$ Jazurke!

Die Prapositionen sind besonders schoer (ich weip vicht urch) ) und die Plural auch, aber an andere sind leiduter Ich studiere die cassetten hören gerter und itingen machen

Auch studiere ich beim Duschen aler es itt sehr schwer, den Walkürenritt singen, und ich singe nur KAKERL+KE! Die Kellerassell, die Kellerassell Könnt nicht schou wautsern, Teil ....

Als kleiner Beitrag zum Thema 'Fremdsprachenpolitik der Junta' die sehr bezeichnende Meinung eines Schülers (Pepe, 40 Jahre, Erster Kurs Instifuto de Idiomas, Uni Sevilla), die man sehr wohl einem der angesprochen Politiker unter die Nase reiben könnte. 
Ith studiere wann ich (Könne und ich möchte mehr Zeit haben, jerand Fore (Konne) ich Keine Jinuten studieren.

Ein Unterridet ist qut wenn lehrt da; wenn ist es Spap, besser. den Uuterridut wag ich, aber manchival gelien wir sehr schnell.

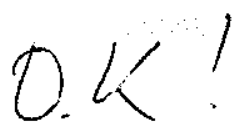

alsiv made gr midut so kouplifient Tepe Roldán

Dazu lässt sich einiges sagen: erstens, dass die Stellen keinesfalls neu geschaffen werden, sondern nur schon bestehende Referendar-Stellen neu besetzt werden. Von Neuausschreibung keine Spur, auch nicht für die attraktiven Dienststellen in Jérez und San Fernando, die wohl immer noch nicht vollständig der Junta unterstellt sind. Also hat sich das Stellenangebot insgesamt nicht erweitert. Zweitens ändert diese Ausschreibung nichts an der traurigen Situation des Deutschen in der ESO. Nur dort könnte sich DaF in der Region wirklich als eine treibende, arbeitsmarktiördernde Kraft entwickeln. Es sieht so aus, als ob die Junta dieses Mal wirklich bereit ist, auf Forderungen seitens der Schulen einzugehen (in der Provinz Sevilla ist dieses Jahr immerhin an drei Schulen zum ersten Mal Deutsch neu eingeführt worden, nachdem der Schulrat den Antrag der Schüler bei der Junta eingereicht hatte). Und wir haben einige Hoffnungen, mit gezielten Aktionen auf verscheidenen Eben etwas erreichen zu können. Auf der anderen Seite aber wird auch laut, dass die Junta die 'Deutsche Frage' nur auf die EOI abwälzen und sich so die Hände waschen will.
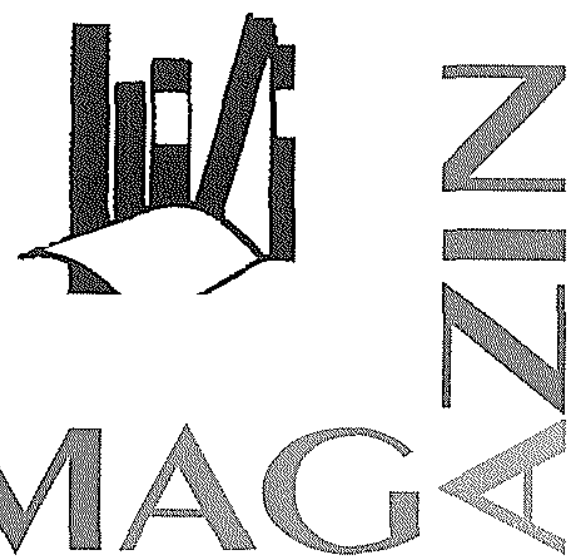